negative (stigma, work load, negative impact on reputation) and the positive impact (detailed review of procedures, implementation of targeted approaches) of the outlier process. Participants felt that sharing experiences of outlying hospitals helps others to improve. They also suggested a 'buddy system' between better and worse performing hospitals. Many highlighted the importance of 'networks' to share experiences, either good or bad, as a vehicle for improving practice.

Discussion The outlier process was generally accepted as a possible mechanism to improve practice. However, participants indicated that effective dissemination is key to ensuring that identifying poor outcomes in some hospitals (e.g. high-risk approach) can stimulate country-wide quality improvement (population approach).

\section{P12 ALCOHOL CONSUMPTION DURING MID-LIFE AND POSTMENOPAUSAL BREAST DENSITY}

V Hailey*, A Britton, S Pinto Pereira. Department of Epidemiology and Public Health, University College London, London, UK

\subsection{6/jech-2020-SSMabstracts. 108}

Background Alcohol consumption and breast density are both established risk factors for breast cancer. Although it has been suggested that the effect of alcohol on breast cancer is via altered breast density, few studies examine whether alcohol consumption at particular life-stages is associated with subsequent mammographic breast density. Average breast density decreases with age and at menopause however women with high alcohol consumption have been shown to have higher breast density. The aim of the study was to examine the association between alcoholic consumption during mid-life and breast density in a population based sample of postmenopausal women.

Methods Data on alcohol consumption and breast density were examined among 833 postmenopausal women from the National Survey of Health and Development, a cohort followed up since their birth in 1946. Mammograms were obtained from routine screening programmes (at approximately age 50 years), from which breast density was calculated. Alcohol intake was self -reporting during mid-life $(36,43 \& 53$ years). Linear regression was used to evaluate the association between weekly grams of alcohol intake at each age and breast density. Regression was used to evaluate the association between alcohol consumption and breast density. This was then adjusted for body mass index (BMI), a known confounder. Then adjusted for BMI and additional confounders; parity, age at first child, age at menstruation, smoking status, physical activity, social status. Age at mammogram and menopause status were constant for all women, therefore no adjustment necessary.

Results In unadjusted analysis a unit increase in weekly alcohol consumption at age 36, 43 and 53 was associated with $4.1 \%$ $\& 3.4 \%$ increase and $0.3 \%$ decrease in breast density respectively. After adjustment for BMI, association remained age 36 with a $2.7 \%$ increase, and lost age $43 \& 53$. All association was lost when adjusted for potential confounders.

Conclusion A 2017 systematic review by Zimbicki and colleagues found a positive association between high alcohol intake and breast density, with a stronger effect seen in premenopausal women. This study suggests that there is no association between alcohol consumption in mid-life and postmenopausal breast density.

\section{P13 ASSOCIATIONS BETWEEN THE LIFE TRANSITIONS OF EARLY ADULTHOOD AND CHANGES IN FAST FOOD INTAKE}

${ }^{1}$ EM Winpenny ${ }^{*},{ }^{2} \mathrm{M}$ Winkler, ${ }^{3,4} \mathrm{~J}$ Stochl, ${ }^{1}$ EMF van Sluijs, ${ }^{2} \mathrm{D}$ Neumark-Sztainer. ${ }^{1} \mathrm{MRC}$ Epidemiology Unit, University of Cambridge, Cambridge, UK; ${ }^{2}$ School of Public Health, University of Minnesota, Minneapolis, USA; ${ }^{3}$ Department of Psychiatry, University of Cambridge, Cambridge, UK; ${ }^{4}$ Department of Kinanthropology, Charles University in Prague, Prague, Czech Republic

\subsection{6/jech-2020-SSMabstracts. 109}

Background Early adulthood is typically a period of poor diet and rapid weight gain. It is also an age of transition, including changes in social and physical environments which may be associated with changes in health-related behaviours. We examine the association of five life transitions (leaving the family home, leaving full-time education, beginning full-time employment, beginning cohabitation, and becoming a parent) with change in fast food intake.

Methods We used four waves of data from adolescence (mean age 15) through early adulthood (to mean age 31) from the longitudinal, population-based Project EAT study (Minnesota, US). The underlying trajectory of fast food intake was modelled as a latent growth curve. Additional latent intercepts at waves 2, 3 and 4 were included, regressed on the 5 life transitions, to allow for additional effects of experiencing life transitions between waves. All life transitions were included in a single model allowing adjustment for other transitions and the underlying growth curve.

Results Fast food was consumed 1.69 times/week (SE 0.03) at age 15 , and followed a negative quadratic trajectory through early adulthood. Beginning full-time employment and becoming a parent were associated with increases in fast food intake of 0.16 times/week (SE 0.007) and 0.16 times/week (SE 0.004) respectively. Leaving the family home and beginning cohabitation were associated with decreases in fast food intake of -0.18 times/week (SE 0.003) and -0.16 times/week (SE 0.008) respectively. Leaving full-time education was not associated with any change in fast food intake (-0.01 times/week (SE 0.89)).

Conclusion Social transitions in early adulthood contribute to changes in fast food consumption, which may affect dietary intake and long-term health. These findings suggest a further focus on the life transitions of beginning employment and becoming a parent for public health policies and intervention.

\section{P14 POOR ORAL HEALTH AND THE ASSOCIATION WITH DIETARY QUALITY AND INTAKE IN OLDER PEOPLE IN TWO STUDIES IN THE UK AND USA} ${ }^{1} \mathrm{E}$ Kotronia*, ${ }^{2} \mathrm{SG}$ Wannamethee, ${ }^{1} \mathrm{H}$ Brown, ${ }^{2} \mathrm{AO}$ Papacosta, ${ }^{3} \mathrm{PH}$ Whincup, ${ }^{2} \mathrm{LT}$ Lennon,
${ }^{4} \mathrm{M}$ Visser, ${ }^{5} \mathrm{YL}$ Kapila, ${ }^{6} \mathrm{RJ}$ Weyant, ${ }^{1} \mathrm{SE}$ Ramsay. ${ }^{1}$ Population Health Sciences Institute,
Newcastle University, Newcastle Upon Tyne, UK; ${ }^{2}$ Department of Primary Care and
Population Health, University College London, London, UK; ${ }^{3}$ Population Health Research
Institute, St George's University of London, London, UK; ${ }^{4}$ Department of Health Sciences,
Vrije Universiteit, Amsterdam, The Netherlands; ${ }^{5}$ Department of Orofacial Sciences,
University of California, San Francisco, USA; ${ }^{6}$ Department of Dental Public Health, University
of Pittsburgh, Pittsburgh, USA

\subsection{6/jech-2020-SSMabstracts. 110}

Background We investigated the associations of poor oral health with dietary quality and intake in older people. We also examined whether changes in dietary quality can influence the risk of oral health problems. 